\title{
Motion Codec Ant Forensics
}

\author{
Nitin Verma \\ Punjab Technical University, \\ Jalandhar
}

\author{
P S Mann \\ Department of Information Technology
}

\begin{abstract}
This paper designs an anti-forensics technique for video files. In this technique the frames are extracted from the video and extracted frames are analysed by using the total variation and the $\mathrm{Q}$. The relation of the total variation and the $\mathrm{Q}$ is used to get whether particular frame is dithered or not. The simulation is carried out on the video downloaded from the internet and analysed by using the Tp rate, Fp rate etc over the MATLAB.
\end{abstract}

\section{Keywords}

Anti-forensics, video, TV, Q

\section{INTRODUCTION}

Forensic techniques are capable of being deceived, thus preventing altered images from being represented as authentic and allowing forensic examiners to establish a degree of confidence in their findings. Furthermore, it is likely that many anti-forensic operations will leave behind detectable fingerprints of their own[1]. If these fingerprints can be discovered, forensic techniques can be designed to detect the use of anti-forensic operations [2].

\begin{abstract}
The rise of digital images coincided with the widespread availability of image editing software led an image forger to easily alter in a visually realistic manner. Organizations desire some means of identifying image alterations and verifying image authenticity to avoid both embarrassment and legal ramifications. This led to the birth of field of digital image forensics. The primary goal of digital image forensics is the identification of images and image regions which have undergone some form of manipulation or alteration. The common properties of the histograms of unaltered images led to building a model of an unaltered image's pixel value histogram. The methods for detecting generally forms globally and locally applied contrast enhancement, as well as a method for identifying the use of histogram equalization, a commonly used form of contrast enhancement [3].
\end{abstract}

\section{RELATED WORK}

The table 1 shows the previous work done:

Table 1: Related Work

\begin{tabular}{|c|c|c|c|}
\hline [4] & 2003 & $\begin{array}{l}\text { Fridrich, A. Jessica, B. } \\
\text { David Soukal, and A. Jan } \\
\text { Lukáš. }\end{array}$ & $\begin{array}{l}\text { Images can be manipulated with editable software's. } \\
\text { Problem of copy-move forgery identified. } \\
\text { Forged part was enhanced/retouched }\end{array}$ \\
\hline [5] & 2003 & $\begin{array}{l}\text { Lukáš, Jan, and Jessica } \\
\text { Fridrich }\end{array}$ & $\begin{array}{l}\text { Primary quantization matrix identified for double compressed JPEG } \\
\text { images. } \\
\text { DCT ch's identified for double JPEG compressed images. }\end{array}$ \\
\hline [6] & 2007 & $\begin{array}{l}\text { Weiqi Luo, Zhenhua Qu, } \\
\text { Jiwu Huang, Guoping Qiu }\end{array}$ & $\begin{array}{l}\text { Symmetric shape of original JPEG image identified by blocking artifact } \\
\text { characteristics matrix (BACM). } \\
\text { BCAM detect the cropping and re-compressed operations on JPEG. }\end{array}$ \\
\hline [7] & 2008 & $\begin{array}{l}\text { Bayram, Sevinc, } \\
\text { HusrevTahaSencar, and } \\
\text { NasirMemon }\end{array}$ & $\begin{array}{l}\text { Achieve general use block-matching procedure. } \\
\text { Divide the image into overlapping blocks . }\end{array}$ \\
\hline [8] & 2009 & $\begin{array}{l}\text { W. Sabrina Lin, Steven } \\
\text { K. Tjoa, H. Vicky Zhao, } \\
\text { K. J. Ray Liu }\end{array}$ & $\begin{array}{l}\text { Two extrinsic techniques are used ; Watermarking and Fingerprint } \\
\text { Identified the digital image source code forensics via intrinsic fingerprint. }\end{array}$ \\
\hline [9] & 2009 & $\begin{array}{l}\text { Matthew C. Stamm. and } \\
\text { K. J. Ray Liu }\end{array}$ & Digital forgeries are detected by unique statistical fingerprints. \\
\hline [10] & 2010 & $\begin{array}{l}\text { Matthew C. Stamm. and } \\
\text { K. J. Ray Liu }\end{array}$ & $\begin{array}{l}\text { A globally and locally applied contrast enhancement. } \\
\text { Issue of histogram equalization by searching for the identifying features } \\
\text { of each operation intrinsic fingerprint }\end{array}$ \\
\hline
\end{tabular}




\begin{tabular}{|c|c|c|c|}
\hline [11] & 2011 & $\begin{array}{l}\text { Feng, Xiaoying, Ingemar } \\
\text { J. Cox, and GwenaëlDoërr }\end{array}$ & $\begin{array}{l}\text { Detect re-sampled imagery. } \\
\text { Normalized energy density identified within windows of varying size. }\end{array}$ \\
\hline [2] & 2011 & $\begin{array}{l}\text { Matthew C. Stamm. and } \\
\text { K. J. Ray Liu }\end{array}$ & $\begin{array}{l}\text { Identify framework for the removal of quantization fingerprint from an } \\
\text { image transform coefficient }\end{array}$ \\
\hline [12] & 2012 & $\begin{array}{l}\text { Tiziano Bianchi, Alessia } \\
\text { De Rosa, Alessandro Piva, }\end{array}$ & $\begin{array}{l}\text { Statistical test is applied to discriminate between original and forged } \\
\text { region JPEG image. }\end{array}$ \\
\hline [13] & 2012 & $\begin{array}{l}\text { P.Bestagini, M.Fontani, S. } \\
\text { Milani, M. Barni, A. Piva } \\
\text { M. Tagliasacchi, S. } \\
\text { Tubaro }\end{array}$ & $\begin{array}{l}\text { Reconstruct the processing history of a given multimedia signal. } \\
\text { These strategies rely on the fact that nonreversible operations applied to a } \\
\text { signal leave some traces ("footprints") that can be identified and classified } \\
\text { in order to reconstruct the possible alterations that have been operated on } \\
\text { the original source. }\end{array}$ \\
\hline [14] & 2012 & $\begin{array}{l}\text { Manimurugan.S, Athira } \\
\text { B.Kaimal }\end{array}$ & $\begin{array}{l}\text { Prevent a medical image compression history } \\
\text { Unnoticeable forgeries can by detect by estimation, examination and } \\
\text { alteration. }\end{array}$ \\
\hline [15] & 2013 & $\begin{array}{l}\text { Böhme, Rainer, and } \\
\text { Matthias Kirchner }\end{array}$ & $\begin{array}{l}\text { Counter-forensics is first defined in a formal decision-theoretic } \\
\text { framework. This framework is then interpreted and extended to } \\
\text { encompass the requirements to forensic analyses in practice, including a } \\
\text { discussion of the notion of authenticity in the presence of legitimate } \\
\text { processing }\end{array}$ \\
\hline
\end{tabular}

\section{PROPOSED ALGORITHM}

Video based anti forensics, the video consists of various frames and each frame is of order $\mathrm{m} \mathrm{X} \mathrm{n}$ and the QA is an array containing the quality factor of each point. The TV is also an array that contains the total variation of each point. In this process, Key Frames are extracted from Video. The average TV for each key frame is calculated. Then by calculating the mean of the average total variation, the overall total variation is calculated. Similarly the average Q is also calculated. If the change in TV is more than average with the same $\mathrm{Q}$ then the frame is dithered. The process can be understood easily by following algorithm:

\section{Motion Codec Ant forensics}

Here, th is the threshold value; threshold value is the constant value taken for the deviation. It is taken as the $1 / 4$ of the average TV value. Mark bit shows the whether the frame is dithered or not.
1. Frames $=$ Extract (Vedio)
2. $\mathrm{J}=1$
3. For $\mathrm{i}=1$ :size(frames, 3 )
4. If $\bmod (1,5)=0$
5. $\quad \operatorname{Keyframe}(;,:, \mathrm{j})=$ frame $(1)$
6. End if
7. Sum_tv=zeros $(\operatorname{size}($ keyframes, 3$))$
8. Sum_Q=zeros $(\operatorname{size}($ keyframes, 3$))$
9. For $\mathrm{i}=1$ :size(keyframes,3)
10. For $\mathrm{k}=1$ :size(keyframes, 2 )
11. For $\mathrm{l}=1$ :size(keyframes, 1 )
12. Sum_tv(i) $=\operatorname{sum} \_t v(i)+T V(k, 1, i)$
13. Sum_Q(i)=sum_Q(i)+Q(k,1,i)
14. End
15. End
16. Sum_tv(i)=sum_tv/(k*l)
17. Sum_Q(i)=sum_Q/(k*l)
18. End
19. Avtv=sum(sum_tv)/i
20. $\mathrm{AvQ}=\operatorname{sum}($ sum_Q$) / \mathrm{i}$
21. Average $Q=z e r o s(\operatorname{size}($ frames, 3$))$
22. Averagetv $=$ zeros $(\operatorname{size}($ frames, 3$))$
23. For $\mathrm{i}=1$ :size(frames, 3 )
24. For $\mathrm{k}=1$ :size(frames, 2 )
25. For $1=1$ :size(frames, 1 )
26. $\operatorname{Averagetv}(\mathrm{i})=\operatorname{averagetv}(\mathrm{i})+\operatorname{frames}(\mathrm{k}, \mathrm{l}, \mathrm{i})$
27. AverageQ $(\mathrm{i})=\operatorname{averageQ}(\mathrm{i})+$ frames $(\mathrm{k}, \mathrm{l}, \mathrm{i})$
28. End
29. End
30. Averagetv $(\mathrm{i})=\operatorname{averagetv}(\mathrm{i}) /(\mathrm{k} * \mathrm{l})$
31. AverageQ $(\mathrm{i})=\operatorname{averageQ}(\mathrm{i}) /(\mathrm{k} * \mathrm{l})$
32. If average $Q(i)>=\operatorname{avQ} \& \&$ average $Q(i)<=a v Q+$ th
33. If averagetv(i) $>$ avtv + th
34. $\operatorname{Mark}(\mathrm{k}, 1, \mathrm{i})=1$
35. End
36. End
If mark is one then the frame is dithered. 


\section{RESULTS AND DISCUSSIONS}

The implementation of the proposed work is carried out on video downloaded from the internet. The implementation process firstly extract frames from the input video. Few extracted frames are shown in Fig 1.

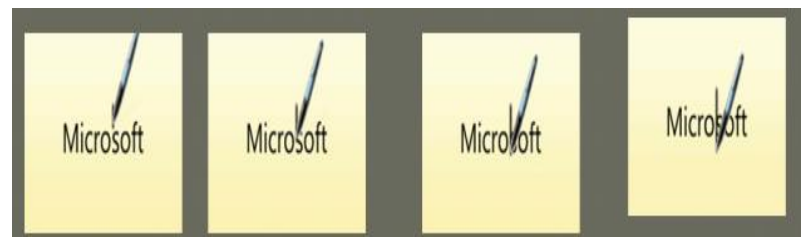

Fig 1: Extracted Frames

Then the algorithm is implemented over the frames and corresponding total variation and histogram are shown in the Fig2.

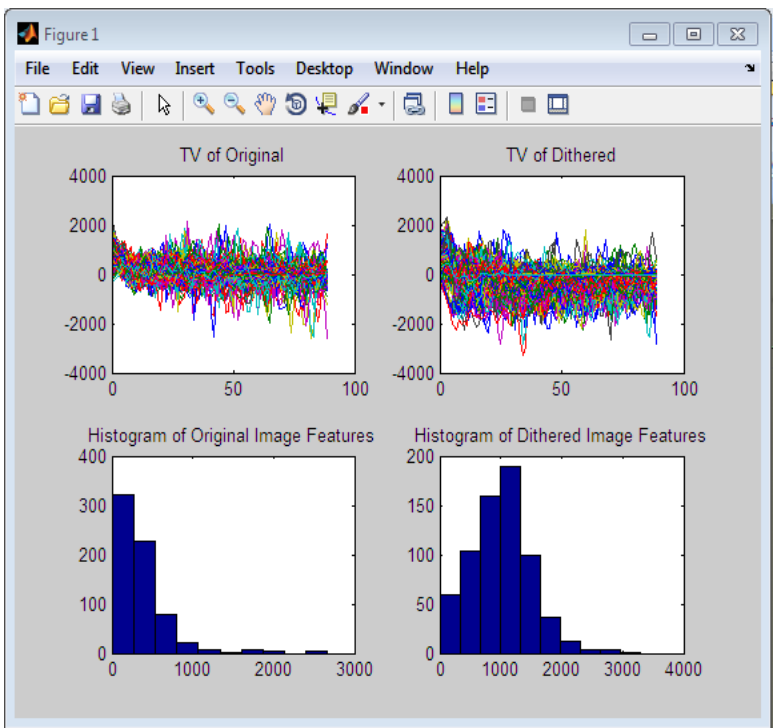

Fig 2: Total Variation of Frame

The parameter evaluation is as follow:

\section{(i) Tp rate $\&$ Fp rate}

The true positive (TP) rate is the fraction of JPEG-compressed images that were correctly reported to be compressed and the false positive (FP) rate is the fraction of uncompressed images that were reported to be compressed.

1. For $\mathrm{i}=1$ :numberoframes

2. $\operatorname{If}(\operatorname{mark}(\mathrm{i}))==1$

3. $\operatorname{If}(\operatorname{status}(\mathrm{i})==1)$

4. Tp++;

5. End

6. Else

7. $\mathrm{Fp}++$

8. end

9. end

10. $\mathrm{tp}=\mathrm{tp} /$ numberofframes

11. $\mathrm{fp}=\mathrm{fp} /$ numberofframes

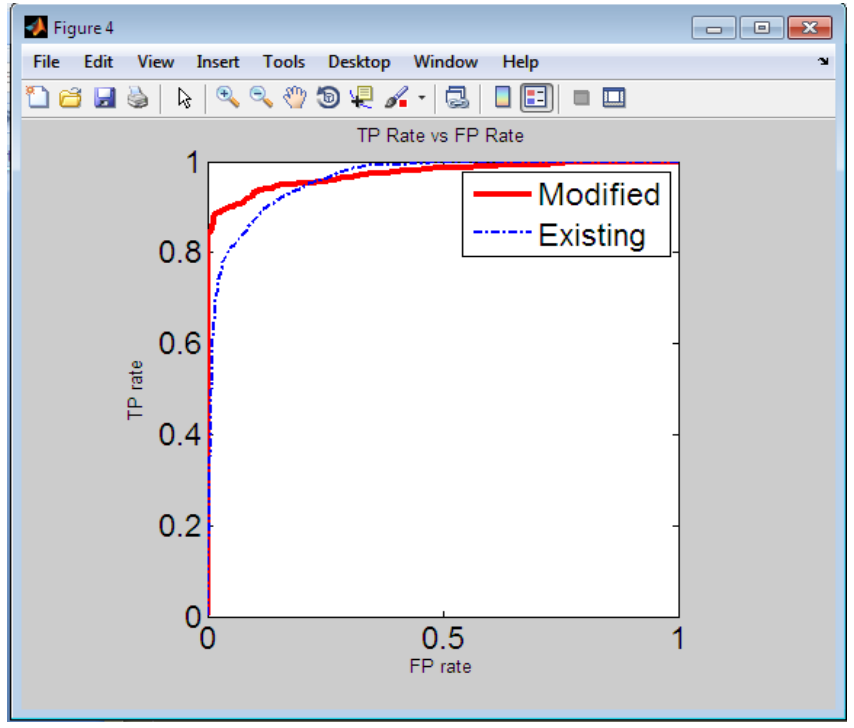

Fig 3: Graph Shows the ROC Curve

(ii) Bias and Standard Deviation

It is the difference between this estimator's expected value and the true value of the parameter being estimated.

Bias can be calculated as

$\epsilon_{\text {bias }}=\frac{1}{T P} \sum_{p \in S}\left(\hat{Q}^{(p)}-Q^{(p)}\right)$

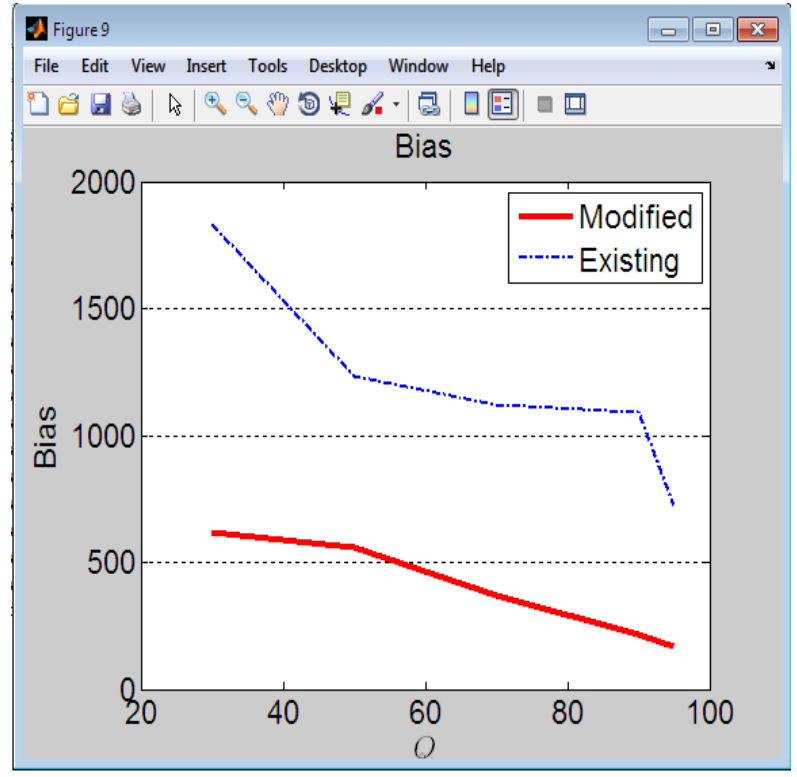

Fig 4: BIAS Comparison

(iii) Standard Deviation

The standard deviation is a measure of how precise the average is, that is, how well the individual numbers agree with each other. It is a measure of a type of error called random error - the kind of error people can't control very well. It is calculated as follows:

$\epsilon_{s d}=\sqrt{\frac{1}{T P} \sum_{p \in S}\left|\hat{Q}^{(p)}-Q^{(p)}\right|^{2}}$

Where $\mathrm{Q}$ is original quality factor and $\hat{Q}$ is estimated quality factor. 
1. for $\mathrm{i}=1$ :noofframes

2. $\quad$ if $(\operatorname{mark}(i)=1$ and $\operatorname{status}(i)=1)$

3. $\mathrm{Q}=$ estimate

4. Sum $=$ sum $+(Q-q)$

5. End

6. Bias $=$ Sum/tp

7. for $\mathrm{i}=1$ :noofframes

8. $\quad$ if $(\operatorname{mark}(i)=1$ and $\operatorname{status}(i)=1)$

9. $\mathrm{Q}=$ estimate

10. $\operatorname{Sum}=\operatorname{sum}+(\mathrm{Q}-\mathrm{q})^{\wedge} 2$

11. End

12. $\mathrm{sd}=\mathrm{sqrt}(\mathrm{Sum} / \mathrm{tp})$

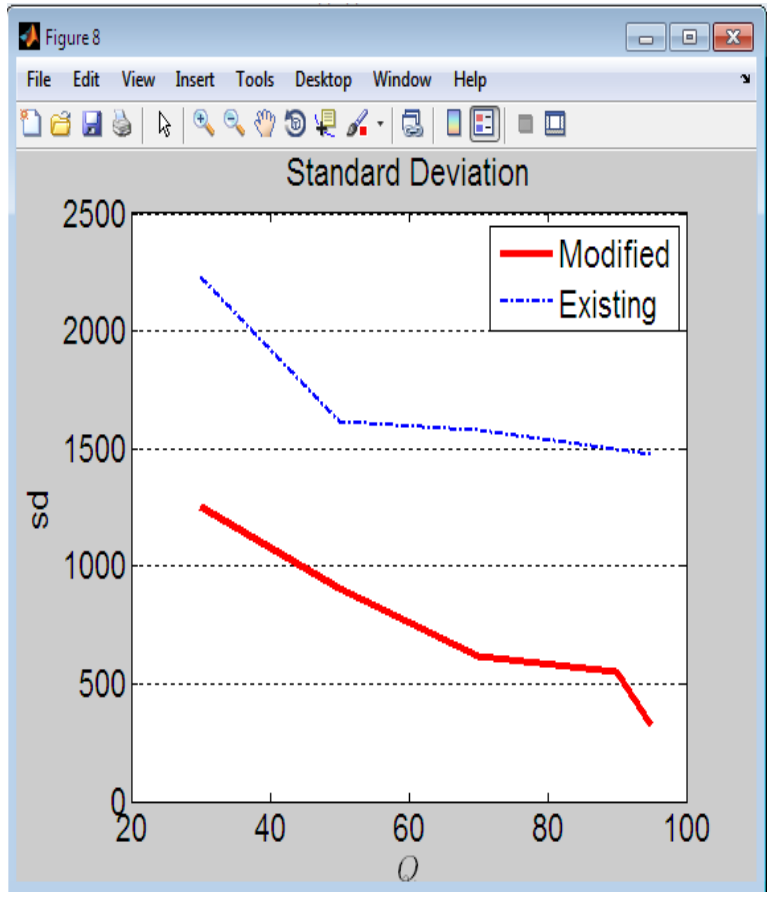

Fig 5: Standard Deviation

(iv) MSE

MSE is computed by averaging the squared intensity of the original (input) image and the resultant (output) image pixels as follow:
1. for $\mathrm{i}=1$ :noofframes
2. for $\mathrm{j}=1$ :row
3. for $\mathrm{k}=1$ :column
4. $\operatorname{sum}=\operatorname{sum}+(\mathrm{o}(\mathrm{u}, \mathrm{k}, \mathrm{i})-\mathrm{d}(\mathrm{j}, \mathrm{k}, \mathrm{i}))^{\wedge} 2$
5. end
6. end
7. end
8. $\operatorname{sum}=\mathrm{sum} / \mathrm{i} * \mathrm{j} * \mathrm{k}$;

MSE can be calculated as:

$M S E=\frac{1}{N M} \sum_{m=0}^{M-1} \sum_{n=0}^{N-1} e(m, n)^{2}$

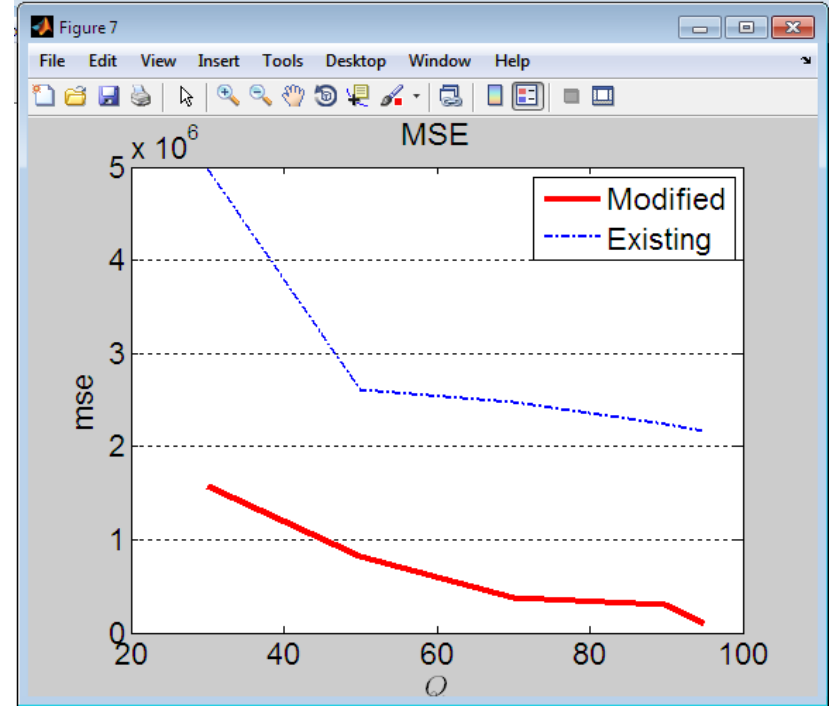

Fig 6: MSE Comparison

(v) PSNR

Peak Signal-to-Noise Ratio (PSNR), Signal-to-noise ratio (SNR) is a mathematical measure of image quality based on the pixel difference between two images. The SNR measure is an estimate of quality of reconstructed image compared with original image. PSNR can be calculated as:

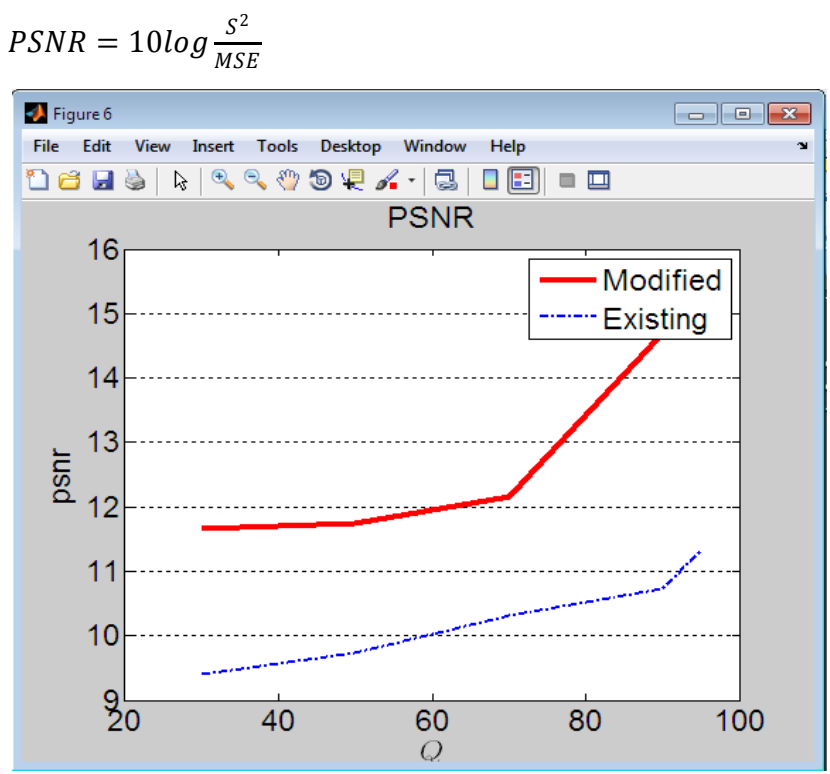

Fig 7: PSNR Comparison

The above analysis shows that the accuracy is improved as compared to the known template results. The Increase in the TP rate and the decrease in the FP rate confirm the benefits of the proposed work.

\section{CONCLUSION AND FUTURE SCOPE}

In this work (Video based anti forensics), the video consists of various frames and each frame is of order $\mathrm{m} \mathrm{X} \mathrm{n}$ and the QA is an array containing the quality factor of each point. The TV is also an array that contains the total variation of each point. In this process, Key Frames are extracted from Video. The average TV for each key frame is calculated. Then by calculating the mean of the average total variation, the overall total variation is calculated. Similarly the average Q is also calculated. If the change in TV is more than average with the 
same $\mathrm{Q}$ then the frame is dithered. The Increase in the TP rate and the decrease in the FP rate confirm the benefits of the proposed work. In future, the work can be extended for the compression anti-forensics for various video and image formats. It can also be extended to extract the hided information in the dithered noise.

\section{REFERENCES}

[1] Matthew C. Stamm, Steven K. Tjoa, W. Sabrina Lin, and K. J. Ray Liu. 2010. Anti-forensics of JPEG compression", In Acoustics Speech and Signal Processing (ICASSP), pp. 1694-1697.

[2] Matthew C. Stamm, Student Member, IEEE, and K. J. Ray Liu, Fellow, IEEE. 2011. Anti-forensics of digital image compression", IEEE Transactions on Information Security, Vol. 6 No. 3, pp. 1050-1065, September.

[3] Stamm, M. C., \& Liu, K. R, 2010. Forensic detection of image manipulation using statistical intrinsic fingerprints, IEEE Transactions on Information Forensics and Security, Vol.5 No.3, pp. 492-506.

[4] Fridrich, A. Jessica, B. David Soukal, and A. Jan Lukáš. 2003. Detection of copy-move forgery in digital images. In Proceedings of Digital Forensic Research Workshop.

[5] Lukáš, Jan, and Jessica Fridrich. 2003 Estimation of primary quantization matrix in double compressed JPEG images. Proc. Digital Forensic Research Workshop.

[6] Weiqi Luo, 2003 A novel method for detecting cropped and recompressed image block, 1-4244-0728-1/07/\$20.00 @IEEE.

[7] Bayram, Sevinc, Husrev Taha Sencar, and Nasir Memon. 2008. A survey of copy-move forgery detection techniques." IEEE Western New York Image Processing Workshop.
[8] W. Sabrina Lin, 2009. Digital Image Source Coder Forensics Via Intrinsic Fingerprints, IEEE Transactions On Information Forensics And Security, VOL. 4, NO. 3.

[9] Matthew C. Stamm 2009 Forensic Detection of Image Tampering Using Intrinsic Statistical Fingerprints in Histograms, Proceedings of 2009 APSIPA Annual Summit and Conference, Sapporo, Japan, October 4-7, 2009.

[10] Matthew C. Stamm 2010.Forensic Detection of Image Manipulation Using Statistical Intrinsic Fingerprints", IEEE Transactions On Information Forensics And Security, VOL. 5, NO. 3, SEPTEMBER.

[11] Feng, Xiaoying, Ingemar J. Cox, and Gwenaël Doërr. 2011. An energy-based method for the forensic detection of Re-sampled images." Multimedia and Expo (ICME), 2011 IEEE International Conference on. IEEE.

[12] Tiziano Bianchi, 2011 Improved Dct Coefficient Analysis For Forgery Localization In Jpeg Images, 9781-4577-0539-7/11/\$26.00 @2011 IEEE.

[13] P. Bestagini , 2012. An Overview On Video Forensics, 20th European Signal Processing Conference (EUSIPCO 2012) Bucharest, Romania, August 27 - 31.

[14] Manimurugan.S (2012) A Tailored Anti-Forensic Approach for Bitmap Compression in Medical Images", IOSR Journal of Computer Engineering (IOSRJCE),ISSN: 2278-0661 Volume 5, Issue 1 SepOct.

[15] Böhme, Rainer, and Matthias Kirchner. 2013. Counterforensics: Attacking image forensics. Digital Image Forensics. Springer New York. 327-366. 